\title{
Improving reliability of power plant equipment due to measurement information redundancy
}

\author{
Kseniia Sapozhnikova ${ }^{1 *}$, Iuliia Baksheeva $^{2}$, and Roald Taymanov ${ }^{1}$ \\ ${ }^{1}$ D.I.Mendeleyev Institute for Metrology, 190005 St.Petersburg, 19 Moskovsky pr., Russia \\ ${ }^{2}$ St. Petersburg State University of Aerospace Instrumentation, 190000 St. Petersburg, 67 Bolshaya \\ Morskaya str., Russia
}

\begin{abstract}
Significant features of using the equipment installed at nuclear, hydraulic and thermal power plants are a multiyear cycle of continuous operation with minimum maintenance works and very high costs necessary to eliminate the consequences of possible crashes. Automatic checking of the state of the most important equipment units can become the optimal decision. In Russia, methods of building intelligent sensors and methods of intellectualization of multichannel measuring systems have been developed. Intellectualization of a measuring system enables to increase the reliability of equipment operation significantly. Examples are given.
\end{abstract}

\section{Intoduction}

Requirements for facilities providing functions of nuclear, hydraulic and thermal power plants have some special features. They are as follows:

- for different equipment, lifetime should lie within the limits from 15 to 60 years;

- a cycle of continuous operation should coincide with the period between repairs and for different equipment, have the duration from 3-4 to 60 years;

- maintenance works should be minimized;

- considering possible hard consequences, emergency risks should be extremely small;

- measuring instruments (MIs) and multichannel measuring systems (MMSs) embedded in such equipment, as a rule, are characterized by non-stationary operation conditions, e.g., significant temperature variations, vibration within a wide frequency and amplitude range, high level of radiation (in case of nuclear power plant), etc.

As a result, MIs and MMSs designed for operation at electric power plants must be characterized by such a distinctive property as very high reliability, in particular, metrological reliability, which can provide high trustworthiness (credibility) of measurement information entering automatic control systems of the plants.

The above-mentioned requirements are much higher than the requirements for MIs designed for wide application. The fact that about $12 \%$ of usual MIs coming for calibration have an error that exceeds permissible limits, illustrates this statement. Such an error originates during a calibration interval that typically is equal to 1-2 years. Herewith, up to $60 \%$ of damages are caused by the errors arisen in the course of operation.

${ }^{*}$ Corresponding author: author@email.org 
In practice, it is impossible to guarantee fault-free operation of devices during many years by improving only their manufacturing technology.

Another method proposed in [1] and developed in much more details later [2-4] was borrowed from evolution. It enables providing the lifetime of biological objects, e.g., human, corresponding to the required lifetime. The essence of the method implies that the system under development is characterized by redundancy, which automatically allows:

- revealing malfunctions and failures;

- signaling to the automatic control system that some malfunction or failure has happened in order to provide making decisions;

- correcting malfunctions with the help of additional (redundant) components.

It is characteristic that in both biological and technical systems, changes are accumulated, which increase the risk of inacceptable disturbances of system operation over time. If we speak about a human, he/she feels that his/her specific sensor system, e.g., sight or hearing (it can also be attributed to any physiological system, e.g., digestion system) starts working worse. Then he/she makes a decision on the method of recovering (e.g., to have a rest or undergo medical treatment). In many cases, he/she gets well or starts using glasses or a hearing aid.

After the proposed method had proved its efficiency, it was included in the Russian national standards $[5,6]$ and called "a metrological self-check". In these standards, sensors and other MIs and MMSs provided by the metrological self-check (MSC) are called "intelligent" ones.

Metrological self-check is an automatic check of the metrological "health" of a MI or MMS in the course of its operation, which is carried out using an accepted reference value generated with the help of an additional (redundant) embedded instrument (a measuring transducer or material measure) or an additional parameter of an output signal. The term "accepted reference value" corresponds to the same term given in [7] and the term "reference quantity value" according to [8]. Monitoring changes of metrological characteristics from their values fixed in the course of previous calibration is the core for the MSC organization. The MSC specifies two versions of its realization using:

- an embedded element (redundant measuring transducer or material measure) of a higher accuracy (metrological direct self-check);

- a reference value of a diagnostic parameter (metrological diagnostic self-check).

Direct self-check reminds the conventional procedure of calibration. The metrological reliability of the embedded material measure or measuring transducer should be significantly higher than that of the MI or MMS under4 checking. The direct MSC can be efficiently applied for measuring electric quantities, but for non-electric ones, it should be used only in some rare cases.

The diagnostic parameter does not characterize the whole uncertainty, but only its critical (dominating) component or component that can grow quickly in the course of operation. With regard to measurements of non-electric quantities, the metrological diagnostic self-check has some advantages.

The method of the metrological diagnostic self-check has enabled the authors of the present paper to demonstrate the possibilities for achieving required improvement of the operation reliability of nuclear and hydraulic power plant equipment.

\section{Sources of information redundancy in power plant equipment}

In MIs or MMSs designed for enhancing the reliability of equipment, the redundancy should be applied in order to organize the MSC.

Redundancy can be of a structural (space), time (frequency), or functional type. 
Developing MIs and MMSs, engineers can reveal redundancy in the equipment or introduce it artificially. The requirement for the redundancy is a rather high stability of a ratio applied as the diagnostic parameter while organizing the self-check. In [2, 9], an intelligent MI designed for measuring the position of a nuclear rod in a nuclear reactor of NPP is described. Its lifetime without calibration corresponds to several decades.

Below, the opportunities for development of intelligent MMSs supporting the energetic equipment reliability enhancement, will be considered in the context of hydroelectric power plant. An intelligent MS intended for monitoring the state of fastening assemblies of the turbine unit cover to its casing, will be considered as an example [10]. Usually, all the power plants where water, steam, or gas as energy carrier provides rotation of a rotor, applies turbines. In the present case, a hydraulic turbine is under consideration, but it does not matter with regard to the methods used.

The cover is fixed at the turbine casing by 80 bolts with screw nuts that are located along the cover circumference. Changing water pressure and vibrations caused by the turbine rotation, lead to bolt stretching, as a result vibration grows which can result in serious crash. To warn in advance about impermissible weakening of cover fixation is a guarantee of safety.

MIs can measure a force pressing a screw nut to a casing, displacement of the nut in relation to the casing, etc. Periodic calibrations of corresponding MIs provide the trustworthiness of measurement results. However, taking into account that sensors embedded in such assemblies should operate under harsh environment, calibration laboratories basing on test results, limit the calibration interval to one year, which is unacceptable economically. The thing is that the required cycle of continuous turbine operation should be equal to 4 years.

The Russian standards $[5,6]$ enable increasing significantly the calibration interval in an intelligent MMS even if it applies sensors with 1-year calibration interval. Therefore, the task comes down to developing an intelligent MMS based on available sensors.

At the first stage, to apply force sensors with quartz resonators using surface acoustic waves was proposed. Their advantage is that they do not require any electric power supply. As a rule, they have acceptable stability and noise immunity of signals. To organize the necessary redundancy, special load washers (LW) were developed. Each LW contains three sensitive elements with quartz resonators mentioned above, which are connected with data processing unit by high frequency cables. Thus, the LW is a MMS.

A set of 8 LWs mounted under nuts on the bolts fastening the cover was installed at one of the Russian hydraulic power plants. Figure 1 shows typical signals of three measuring channels of the LW registered at the hydroelectric power plant.

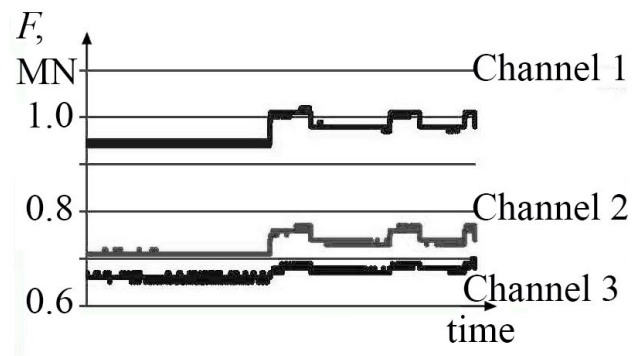

Figure 1. Typical signals of three measuring channels of the LW ( $F$ is compression force)

Analysis has shown that the channel measuring the maximum compression force is the most stable. The MSC algorithm includes cycles of the following main operations: 
- data acquisition from all the channels and recording of measured pressing force values in these channels;

- determination of the channel number with the maximum pressing force value for each LW (This channel for the given LW is assigned as a reference one);

- starting from the second cycle, determination and recording of the pressing force value changes in the channels with the maximum pressing force;

- detection of the LW that is characterized by the best stability (This LW and the corresponding channel are assigned as the reference ones for the set as a whole);

- calculation of the correlation coefficients between the output signal of the channel assigned as the reference one for the whole set and the signals in the reference channels of the rest LWs;

- calculation of the differences between the current correlation coefficients mentioned above and the coefficients determined within the previous cycle (If such a difference exceeds some given limit, then a signal informing about a possible defect is formed);

- analysis of the trends of compression force variation in reference channels and those of correlation coefficients (If both compression forces decrease, at least, in a few nearby channels and variations of the correlation coefficients in the same channels increase, this fact indicates that fastening is weakening. If necessary, an alarm signal can be generated).

The experience of LW operation has shown that force sensors based on quartz resonators are not satisfactory reliable, in particular, from the metrology point of view. Meanwhile, the efficiency of using correlations between the nut compression forces is not sufficient for organizing the MSC.

This circumstance has resulted in the development of an intelligent MMS using displacement sensors.

In a new intelligent MMS, like in the system considered above, the sensors are installed at each tenth bolt. However, the sensors are single-channel tactile linear displacement sensors, their output being a code signal. The displacement sensors were chosen from the sensors that are mass-produced by a Russian company that provides the requirements of energetics. According to the specification, the sensor uncertainty is less that $5 \mu \mathrm{m}$ within the range $\pm 3 \mathrm{~mm}$.

To reveal redundant information, a postulate has been accepted that the turbine cover is stiff. This postulate enables self-checking of the fastening assemblies based on information that when the pressure under the cover causes stretching the bolts, bolt displacements correlate with each other. This effect takes place regardless whether the cover moves in parallel to an original fixing plane or with a slope.

To develop the MSC algorithm, at the initial stage of sensor operation, the decision was made to assess the probability of potentially dangerous failures of sensors, which could take place if they operate at a hydraulic unit. Obtained data should enable improving the sensor design in the future by providing the maximum sensor reliability.

The analysis of the design has shown that if sensors are installed into fastening assemblies, dynamic loads on the turbine cover can lead to arising forces directed perpendicular to the axis of the sensor. In its turn, this fact can cause increased friction and "sticking" of a sensor moving parts. As a result, in the algorithm, calculation of the number of temporal "sticking" and "detaching" (i.e., failures arising occasionally and disappearing without staff intervention) is carried out.

To improve the trustworthiness of measurement information and efficiency of hardware resources, the algorithm provides a preliminary processing of data coming from the measuring channels with $2 \mathrm{~Hz}$ frequency.

The preliminary data processing includes a "thinning" of the original data set and data smoothing of "thinned" data in non-crossing time windows by calculation of medians. The 
median evaluation allows removing outliers and provides the higher accuracy of calculations taking into consideration available hardware resources.

The value of the "thinning" data frequency was chosen by simulating the procedures based on signal records obtained from analogues turbine units.

The simulation method allowed also carrying out justification of smoothing window duration and the number of necessary windows to accumulate information required for detection of the sensor "sticking". Data on the average deviation of signals from their median, were used as a criterion.

The algorithm also determines a short-term failures such as bidirectional signal variation and big outliers in nearby windows, reveals defective sensors as well as evaluates the danger level in case of weakening the fastening assemblies.

The results of data processing during the whole period of turbine operation, enable determining measurement uncertainty drift. Current results of the MSC are recorded into a data logger. They include information on:

- failures detected,

- alerts and forecasts related to operation of the MMS,

- slope of the turbine unit cover,

- uncertainty drift and self-correction made.

The MSC provides confidence in the trustworthiness of measurement results and allows faulty sensors to be detected. It gives grounds to carry out periodic calibrations of sensors, only in the course of planned shutdowns of turbine units. Therefore, as a rule, metrological maintenance procedures can be performed not so often as it would be necessary for nonintelligent MMSs.

To verify the algorithm efficiency, virtual tests of the developed MMS were carried out. 15-day tests applied records of the signals obtained from similar sensors embedded in fastening assemblies. The records corresponded to typical modes of the turbine unit operation.

\section{Conclusion}

The considered intelligent multichannel measuring system realizes the metrological selfcheck concept developed at the D.I. Mendeleyev Institute for Metrology, Russia, and described in two Russian national standards, on an industrial scale.

The experience gained in this development can be useful while building measuring systems of the Industry 4.0 generation. In our opinion, this experience should be used in the preparation of international standards related to multichannel measuring systems, reliability of which is particularly important.

\section{References}

1. R. Taymanov, K. Sapozhnikova Proc. of XVII IMEKO World Congr., Dubrovnik, 1094-1097 (2003)

2. K. Sapozhnikova, R. Taymanov Nuclear power - control, reliability and human factors, ed. P. Tsvetkov (InTech, 2011) 3-26

3. K. Sapozhnikov, A. Pronin, I. Druzhinin, R. Taymanov 14th IMEKO TC10 Workshop on technical diagnostics 181-186 (2016)

4. R. Taymanov, K. Sapozhnikova, Chapter 1 Smart sensors and MEMS for industrial applications ed. S. Nihtianov, A.L. Estepa (Woodhead Publishing, Elsevier Limited, 2018) 1-22 
5. GOST R 8.673-2009 Intelligent sensors and intelligent measuring systems. Basic terms and definitions (Standartinform, Moscow, 2010) (in Russian)

6. GOST R 8.734-2011 Intelligent sensors and intelligent measuring systems. Methods of metrological self-checking (Standartinform, Moscow, 2011) (in Russian)

7. ISO 5725-1:1994 Accuracy (trueness and precision) of measurement methods and results - Part 1: General principles and definitions (1994)

8. International vocabulary of metrology - basic and general concepts and associated terms (VIM) 2008 version with minor corrections (BIPM, JCGM 200, 2012)

9. R. Taymanov, K. Sapozhnikova, I. Druzhinin Nuclear Plant Journal 25 2, 45-47 (2007)

10. R. Taymanov, K. Sapozhnikova, I. Danilova, I. Druzhinin. XXI IMEKO World Congr. 5 p. (2015) 